\title{
On the Vibration Modes of the Air-Water Interface in the Presence of Surface Films.
}

P. L. Ghia and P. Trivero

Istituto di Fisica Generale dell'Università - Torino

Istituto di Cosmogeofisica del C.N.R. - Torino

(Nuovo Cimento C, 11, 305 (1988))

Equations (9b) and (10b) should read as follows:

$$
\begin{aligned}
& \eta_{\mathrm{d}}=\varepsilon_{0} \frac{\tau_{\mathrm{B}}}{\left(1+2 \tau_{\mathrm{B}}+2 \tau_{\mathrm{B}}^{2}\right) \omega}, \\
& \eta_{\mathrm{d}}=\varepsilon_{0} \frac{-\tau_{\mathrm{i}}}{\left(1-2 \tau_{\mathrm{i}}+2 \tau_{\mathrm{i}}^{2}\right) \omega} .
\end{aligned}
$$

